\title{
Electron-ion interaction effects in attosecond time-resolved photoelectron spectra
}

\author{
C.-H. Zhang* and U. Thumm \\ Department of Physics, Kansas State University, Manhattan, Kansas 66506, USA \\ (Received 12 February 2010; revised manuscript received 13 August 2010; published 6 October 2010)
}

\begin{abstract}
Photoionization by attosecond extreme ultraviolet (xuv) pulses into the laser-dressed continuum of the ionized atom is commonly described in strong-field approximation, neglecting the Coulomb interaction between the emitted photoelectron (PE) and the residual ion. By solving the time-dependent Schrödinger equation, we identify a temporal shift $\delta \tau$ in streaked PE spectra, which becomes significant at low PE energies. Within an eikonal approximation, we trace this shift to the combined action of Coulomb and laser forces on the released $\mathrm{PE}$, suggesting the experimental and theoretical scrutiny of their coupling in streaked PE spectra. Further, we examined the effect of initial state polarization by the laser pulse on the xuv streaked spectrum.
\end{abstract}

DOI: 10.1103/PhysRevA.82.043405

PACS number(s): 42.50.Hz, 42.65.Re, 79.60.-i

\section{INTRODUCTION}

Using an extreme ultraviolet (xuv) attosecond pulse to photoemit electrons from gaseous or solid targets into the electric field of a synchronized delayed femtosecond (fs) infrared (ir) laser pulse provides a powerful tool for investigating ultrafast electron dynamics by recording ir-laser-streaked xuv photoelectron (PE) spectra [1]. For strong laser fields and sufficiently fast PEs, streaked PE spectra are conveniently described in the strong-field approximation (SFA) [2], that is, by ignoring the interaction of the residual ion with the released PE. In this case, subject only to the ir-laser electric field, the propagation of PEs can be described in terms of analytically known "Volkov" states [3]. This leads to a delay-dependent energy shift, $\delta E_{\mathrm{COE}}^{\mathrm{SFA}}(\tau)=-k A_{L}(-\tau)$, of the center of energy (COE) $E_{\mathrm{COE}}=\omega_{X}-\left|\varepsilon_{B}\right|$ in the PE spectrum, where $\omega_{X}$ is the xuv-pulse central frequency, $\varepsilon_{B}$ the binding energy in the initial bound state, $k$ the PE asymptotic momentum, and $A_{L}$ the vector potential of the ir pulse. We use atomic units except where stated otherwise and define the delay $\tau$ between the centers of the xuv and the ir pulse as positive if the xuv pulse precedes the ir pulse.

The interpretation of sub-fs temporal shifts in streaked PE spectra is a matter of current debate. For example, the recent measurement [4] of a relative delay of $\approx 110 \pm 70$ as between the ir-streaked xuv photoemission from localized $4 f$ core levels and delocalized conduction-band (CB) states of a W(110) surface was understood in the original Ref. [4] and a subsequent theoretical work [5] as the difference $\delta t_{\mathrm{CB}-4 f}=t_{\mathrm{CB}}-t_{4 f}$ between the arrival times of $\mathrm{CB}$ and $4 f$ core PEs at the surface. This interpretation is based on the assumption that the ir pulse does not penetrate the surface, such that $\mathrm{CB}$ and $4 f$ electrons that are released at the same time $-\tau$ by the absorption of an xuv photon get streaked only upon arrival at the surface, producing the COE shifts $\delta E_{\mathrm{COE}}(\tau)=-k A_{L}\left(-\tau+t_{\mathrm{CB}}\right)$ and $-k A_{L}\left(-\tau+t_{4 f}\right)$, respectively, in the PE spectra. According to this two-step explanation (photorelease followed by streaking), the total emission probability $P_{\mathrm{tot}}=\int d E P(E, \tau)$ from a given initial state would not depend on $\tau$.

\footnotetext{
*chhzhang@ksu.edu
}

In contrast, our analysis of experimental streaked photoemission data for a tungsten [4] and a rhenium [6] surface indicates that $P_{\mathrm{CB}}(\tau)$ oscillates with $A_{L}(\tau)$, with an amplitude of $\approx 10 \%$ of the average value. Furthermore, the continuity of the wave function and its derivative at the surface implies that an intense fs ir pulse affects the PEs inside the solid, even if the ir electric field is prohibited from penetrating the surface [7]. We have shown that this observed temporal shift can be reproduced within the SFA and interpreted it as an interference effect in the emission from different lattice sites [3], observing that the SFA cannot account for relative temporal shifts in the emission from different levels of isolated atoms. A classical transport simulation including the effect of (in)elastic collisions of released PEs with tungsten cores on the propagation of $\mathrm{PE}$ inside the solid leads to $\delta t_{\mathrm{CB}-4 f}=33$ as in [8]. Thus, different models [3-5,8,9] deviate strongly with regard to the assumed attenuation of the streaking ir electric field $E_{L}(t)$ inside the solid, ranging from no penetration into the surface [5] to penetration depths of 30 [3] and 85 [8] layer spacings, or larger than the electron mean free path [9]. Detailed modeling of the (relative) delay in photoemission from metal surfaces is further complicated by the complex band structure and the ensuing difficulty in assigning a group velocity to the motion of $\mathrm{PE}$ wave packets inside the dispersive conduction band [4], surface charge accumulation, and the general concern that static band-structure calculations and the assumption of an instantaneous plasmon response (i.e., static image charge interactions) are invalid at the attosecond time scale. These shifts may be of particular importance in the interpretation of streaking spectra for complex targets, such as metals, and emphasize the need for more detailed studies of streaked photoemission spectra.

It is of fundamental importance to first understand all contributions to this temporal shift for simple systems. In this work, we focus on the effect of simultaneous ir-laser pulse and Coulomb interactions on streaked photoemission spectra from the prototypical ground state of a one-dimensional (1D) hydrogen atom. This Coulomb-laser (CL) coupling effect was first investigated by Kroll and Watson [10] in their study of laser-assisted atomic scattering. It also affects the spectra of high harmonic generation, multiphoton ionization, and laser-assisted xuv photoionization. For example, the Coulomb interaction causes the xuv streaked PE spectra to be right-left asymmetric [11]. In RABITT (Reconstruction of 
Attosecond Beating by Interference of Two Photon Transition) measurements, these simultaneous ir- laser pulse and Coulomb interactions induce a so-called atomic phase which shifts sideband intensities as a function of the delay between the xuv pulse train and the ir pulse [12-15].

In this work, we demonstrate, numerically and analytically, how the coupling of the ir-laser pulse and the final-state Coulomb interaction of the PE with the residual ion gives rise to a significant temporal shift $\delta \tau$ in the COE of streaked PE spectra with respect to those approximated in SFA. As we will show, inclusion of this CL coupling alters both the amplitude and the phase of the COEs in streaked PE spectra, leading to a COE shift, $\delta E_{\mathrm{COE}}^{\mathrm{CL}}(\tau)=-K A_{L}(\tau-\delta \tau)$, with an oscillation amplitude $K>k$. Thus, $\delta \tau$ and the streaking amplitude ratio $K / k$ (i) help to reveal details of the PE dynamics including the combined interaction of Coulomb and laser forces and (ii) converge to their SFA limits, 0 and 1, respectively, at sufficiently higher PE energies.

We numerically solve the time-dependent Schrödinger equation (TDSE), including the electron-proton interaction, and then compare our results for $P(E, \tau)$ with SFA and eikonal approximation (EA) calculations for a large range of PE kinetic energies. We find temporal shifts $\delta \tau$ of more than 50 as, strongly dependent on $\omega_{X}$. Figures $1(\mathrm{a})$ and $1(\mathrm{~b})$ show the TDSE ir-streaked PE spectra $P(E, \tau)$ for Gaussian xuv pulses of length $\tau_{X}=300$ as, with $\hbar \omega_{X}=90$ and $25 \mathrm{eV}$, respectively. The ir pulse is also assumed to be a Gaussian and has a peak intensity of $I_{L}=2 \times 10^{12} \mathrm{~W} / \mathrm{cm}^{2}$, a carrier frequency $\omega_{L}=1.6 \mathrm{eV} / \hbar$, and a pulse length $\tau_{L}=5 \mathrm{fs}$. These spectra are shifted by $\delta \tau=60$ as, which becomes apparent in the corresponding $\mathrm{COE}$ shifts $\delta E_{\mathrm{COE}}(\tau)$ in Fig. 1(c). The solid curves in Figs. 1(d) and 1(e) show $\delta \tau$ and the ratio $K / k$ of streaking-oscillation amplitudes for a large range of $\omega_{X}$. Within an EA approach $[11,16,17]$, we can trace (details are given later) this $\omega_{X}$-dependent temporal shift and the oscillation amplitude enhancement to the CL coupling in the
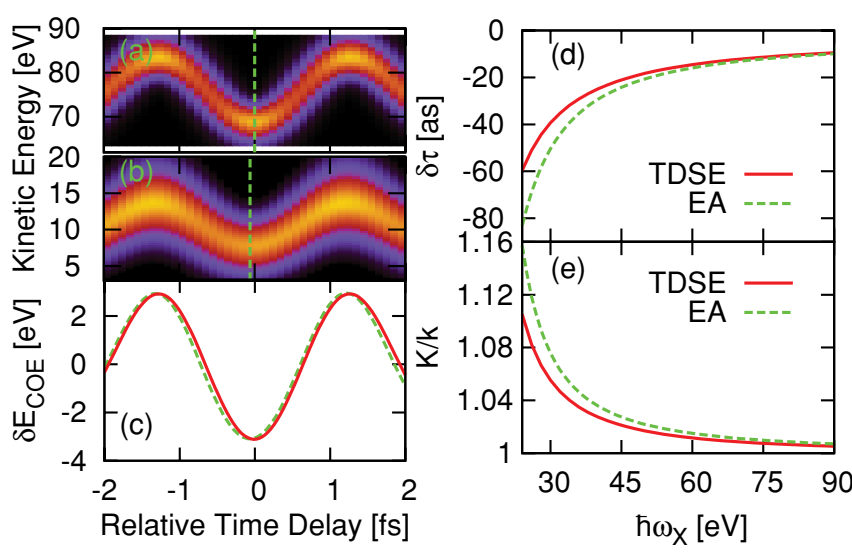

FIG. 1. (Color online) Streaked photoemission from 1D model hydrogen atoms. TDSE calculations for xuv pulses with (a) $\hbar \omega_{X}=$ $90 \mathrm{eV}$ and (b) $\hbar \omega_{X}=25 \mathrm{eV}$. (c) Corresponding centers of energy $\delta E_{\mathrm{COE}}(\tau)$ for $\hbar \omega_{X}=90 \mathrm{eV}$ (solid line) and $\hbar \omega_{X}=25 \mathrm{eV}$ (dashed line). To facilitate the identification of the relative temporal shifts $\delta \tau, \delta E_{\mathrm{COE}}\left(\tau, \hbar \omega_{X}=90 \mathrm{eV}\right)$ is normalized to the $\hbar \omega_{X}=25 \mathrm{eV}$ result. (d) $\delta \tau$ and (e) oscillation amplitude relative to the SFA for TDSE (solid line) and eikonal approximation (dashed line) calculations.
PE final state [dashed curves in Figs. 1(d) and 1(e)]. $\delta \tau$ and $K / k$, for the TDSE and EA calculations, converge at large $\omega_{X}$ to their respective SFA limits, $\delta \tau=0$ and $K / k=1$, due to the diminishing influence of the residual ion's Coulomb force at increasing PE energies. Thus, $\delta \tau$ and $K / k-1$ are measures for the combined action of the Coulomb and laser force on the PE relative to the action of the ir-laser force alone. Since $\delta \tau<0$, the attractive Coulomb force does not delay the PE emission, as one might intuitively expect. We also note that $K>k$ reveals a Coulomb-enhancement effect that is reminiscent of the Coulomb potential's infinite range leading to well-understood "Coulomb cusps" in energy-differential collision-induced PE spectra [18].

This article is organized as follows. In Sec. II, we present numerical results based on the TDSE. In Sec. III, we adopt an EA to take into account the simultaneous ir and Coulomb interactions of the PEs and compare our EA and TDSE results. In Sec. IV, we examine the effect of the polarization of the initial state on the streaked spectrum. We conclude in Sec. V. In the Appendix, we show that, within the EA, the atomic phase obtained in RABITT is identical to the relative temporal shift in the streaked PE spectrum.

\section{TIME-DEPENDENT SCHRÖDINGER EQUATION FOR STREAKING}

The exact wave function of the 1D model atom interacting with the ir and xuv pulse is determined by the TDSE (in the length gauge)

$$
i \frac{\partial}{\partial t} \Psi(x, t)=\left[-\frac{1}{2} \frac{d^{2}}{d x^{2}}+U(x)+V(x, t)\right] \Psi(x, t),
$$

where $U(x)$ is the Coulomb potential, $V(x, t)=x\left[E_{L}(t)+\right.$ $\left.E_{X}(t)\right]$ the interaction with the ir and xuv pulse, and $E_{L(X)}$ the electric field of the ir (xuv) pulse. Assuming single-photon ionization in a sufficiently weak xuv pulse, and after splitting the exact wave function for the atom in the combined xuv and ir electric fields according to $\Psi(x, t)=\psi_{g}(x, t)+\delta \psi(x, t),(1)$ can be replaced by two coupled equations [19]:

$$
\begin{aligned}
i \frac{\partial}{\partial t} \psi_{g}(x, t)= & {\left[-\frac{1}{2} \frac{d^{2}}{d x^{2}}+U(x)+x E_{L}(t)\right] \psi_{g}(x, t) } \\
i \frac{\partial}{\partial t} \delta \psi(x, t)= & {\left[-\frac{1}{2} \frac{d^{2}}{d x^{2}}+U(x)+x E_{L}(t)\right] \delta \psi(x, t) } \\
& +x E_{X}(t+\tau) \psi_{g}(x, t) .
\end{aligned}
$$

Equation (2) determines the evolution (polarization) of the initial state in the ir field and (3) the generation of PE wave packets by the xuv pulse and their evolution in the ir field. The electric fields $E_{L(X)}=-\partial A_{L(X)}(t) / \partial t$ of the ir (xuv) pulses are derived from the vector potentials $A_{L(X)}(t)=$ $A_{L(X), 0} \cos \left(\omega_{L(X)} t\right) e^{-2 \ln 2 t^{2} / \tau_{L(X)}^{2}}$. Since $E_{L(X)}(t \rightarrow \pm \infty)=0$, Eqs. (2) and (3) are subject to the initial conditions $\psi_{g}(x, t \rightarrow$ $-\infty)=\psi(x) e^{-i \varepsilon_{B} t}$ and $\delta \psi(x, t \rightarrow-\infty)=0$. The groundstate initial wave function $\psi(x)$ and energy $\varepsilon_{B}$ are obtained 
from

$$
\varepsilon_{B} \psi(x)=\left[-\frac{1}{2} \frac{d^{2}}{d x^{2}}+U(x)\right] \psi(x) .
$$

We solve (2)-(4) numerically by wave-packet propagation for times $|t| \leqslant 2.5 \tau_{L}$ with a step size $\Delta t=0.2$ on a spatial grid with $|x| \leqslant 2000$ and spacing $\Delta x=0.25$. Assuming free-electron dispersion, $E=\frac{1}{2} k^{2}$, we calculate the ir-assisted xuv photoemission probability,

$$
P(E, \tau)=|\delta \tilde{\psi}(k, \tau, t \rightarrow \infty)|^{2},
$$

and the corresponding $\mathrm{COE}$,

$$
E_{\mathrm{COE}}(\tau)=\frac{1}{2} \int d k|k \delta \tilde{\psi}(k, \tau, t \rightarrow \infty)|^{2} / P_{\mathrm{tot}}(\tau),
$$

where $\delta \tilde{\psi}(k, \tau, t)$ is the Fourier transform of $\delta \psi(x, t)$, and the total emission probability is

$$
P_{\mathrm{tot}}(\tau)=\int d k|\delta \tilde{\psi}(k, \tau, t \rightarrow \infty)|^{2} .
$$

We model the target atom based on the soft-core Coulomb potential,

$$
U(x)=-\frac{1}{\sqrt{x^{2}+a^{2}}},
$$

and adjust the parameter $a=\sqrt{2}$ to the ground-state binding energy $\varepsilon_{B}=-13.6 \mathrm{eV}$ of the hydrogen atom. We refer to the exact solution of (2)-(4) as the "TDSE result" and retrieve the SFA results by ignoring $U(x)$ in (3). The comparison of TDSE and SFA results is shown in Figs. 1(a)-1(c). By dropping the laser interaction $x E_{L}(t)$ in (2), we verified numerically that, for the given parameters, the polarization of this initial state by the ir pulse can be neglected $[9,19]$. The initial-state polarization effect on the streaked xuv PE spectrum is further discussed in Sec. IV.

\section{EIKONAL APPROXIMATION}

To trace the influence of the combined action of the Coulomb potential and ir pulse on the PE, we write the PE wave function as

$$
\psi_{k}(x, t)=a_{k}(x, t) e^{i\left[k+A_{L}(t)\right] x-i k^{2} t / 2+i S_{k}(x, t)},
$$

with a local phase $S_{k}(x, t)$. The real amplitude $a_{k}$ is not important for the present investigation. In SFA, the phase $S_{k}(x, t)$ is given by the Volkov phase,

$$
S_{k}^{\mathrm{SFA}}(t)=k \int_{t}^{\infty} d t^{\prime} A_{L}\left(t^{\prime}\right)
$$

and independent of $x$. In EA, and without the ir field, the phase accumulated by the PE during its propagation in $U(x)$ from location $x$ at time $t$ to the electron detector is calculated along the free-electron classical trajectory $x^{\prime}\left(t^{\prime}, t, x\right)=x+k\left(t^{\prime}-\right.$ t) [16]:

$$
S_{k}^{C}(x)=\int_{t}^{\infty} d t^{\prime} U\left[x^{\prime}\left(t^{\prime}, t, x\right)\right]=\frac{1}{k} \int_{x}^{\infty} d x^{\prime} U\left(x^{\prime}\right) .
$$

In the presence of the ir field, the free-electron classical trajectory is modified by a laser-induced drift,

$$
\Delta x\left(t, t^{\prime}\right)=\int_{t}^{t^{\prime}} d t^{\prime \prime} A_{L}\left(t^{\prime \prime}\right)
$$

to become

$$
x_{L}\left(t^{\prime}, t, x\right)=x^{\prime}\left(t^{\prime}, t, x\right)+\Delta x\left(t, t^{\prime}\right) .
$$

Replacing $x^{\prime}$ with $x_{L}$ in (11), we obtain the CL phase [11,17],

$$
S_{k}^{\mathrm{CL}}(x, t)=\int_{t}^{\infty} d t^{\prime} U\left[x_{L}\left(t^{\prime}, t, x\right)\right],
$$

and an EA to the local phase in (9),

$$
S_{k}^{\mathrm{EA}}(x, t)=S_{k}^{\mathrm{SFA}}(t)+S_{k}^{\mathrm{CL}}(x, t) .
$$

In typical streaking experiments and for this study, the ir intensity $\left(\sim 10^{12} \mathrm{~W} / \mathrm{cm}^{2}\right)$ is low enough for $\Delta x\left(t, t^{\prime}\right)$ being a small deviation from $x^{\prime}\left(t^{\prime}, t, x\right)$. We thus expand $S_{k}^{\mathrm{CL}}$ about $x^{\prime}\left(t^{\prime}, t, x\right)$ and obtain, to first order in $\Delta x\left(t, t^{\prime}\right)$,

$$
S_{k}^{\mathrm{CL}}(x, t)=S_{k}^{C}(x)-\int_{t}^{\infty} d t^{\prime} F\left[x^{\prime}\left(t^{\prime}, t, x\right)\right] \Delta x\left(t, t^{\prime}\right),
$$

with the Coulomb force

$$
F\left[x^{\prime}\left(t^{\prime}, t, x\right)\right]=-\frac{\partial U\left[x^{\prime}\left(t^{\prime}, t, x\right)\right]}{\partial x^{\prime}} .
$$

The first term in (16) is the laser-free eikonal Coulomb phase. This term is independent of time as we explicitly indicate in (11). As we will show, this phase does not induce any temporal shift in the streaked xuv spectrum but changes the transition probability. The second term in (16) includes Coulomb scattering of the PE while it absorbs or releases ir photons [17]. It is proportional to the ir vector potential and causes a temporal shift in the streaked spectrum.

The numerical results for (16) in Fig. 2(a) resolve the spatial contributions to the CL coupling phase $S_{k}^{\mathrm{CL}}(x, t)-S_{k}^{C}(x)$. Keeping in mind that the EA is designed for short PE de Broglie wavelengths [16], we confirmed, by comparison with full TDSE results (not shown), that $S_{k}^{\mathrm{CL}}(x, t)$ remains appropriate down to $k=1$, that is, a PE energy of $\approx 14 \mathrm{eV}$ [11]. This supports the validity of the EA for the range of PE kinetic energies in Figs. 1 and 2.

The transition amplitude for xuv photoemission from the initial state $\psi_{i}$ to the final state $\psi_{k}$,

$$
T_{k}(\tau)=-i \int d t\left\langle\psi_{k}^{*}(t)\left|x E_{X}(t+\tau)\right| \psi_{i}(t)\right\rangle,
$$
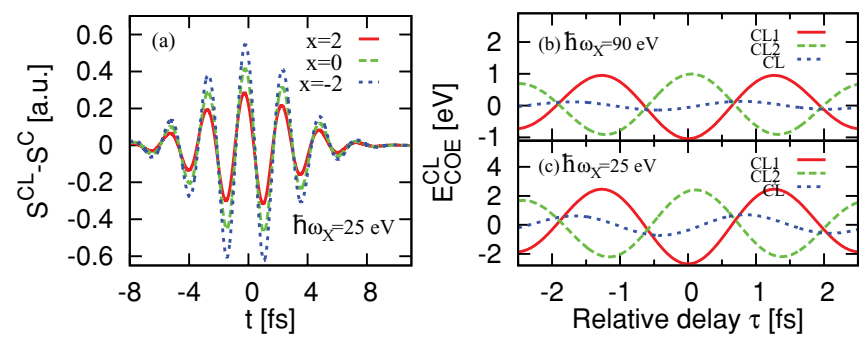

FIG. 2. (Color online) (a) Time evolution of the CL coupling phase (see text). Contribution to the PE streaking $\delta E_{\mathrm{COE}}^{\mathrm{EA}}(\tau)$ at $x=0$ due to final-state CL coupling at (b) $\hbar \omega_{X}=90 \mathrm{eV}$ and (c) $\hbar \omega_{X}=$ $25 \mathrm{eV}$. 
provides the PE probability $P\left(E=k^{2} / 2, \tau\right)=\left|T_{k}(\tau)\right|^{2}$ as an alternative to (5). Neglecting the laser distortion of the initial state [using $\psi_{i}(x, t) \approx \psi_{i}(x) e^{-i \varepsilon_{B} t}$ ] and employing the EAapproximated PE wave function for $\psi_{k}(x, t)$, we obtain

$$
\begin{aligned}
T_{k}^{\mathrm{EA}}(\tau)= & -i \int d t d x a(x, t) e^{-i\left[k+A_{L}(t)\right] x} x \psi(x) \\
& \times E_{X}(t+\tau) e^{-i S_{k}^{\mathrm{EA}}(x, t)} e^{-i\left(\varepsilon_{B}-k^{2} / 2\right) t} .
\end{aligned}
$$

The COE of the spectrum for a free PE would be $E_{\mathrm{COE}}=$ $k^{2} / 2=\omega_{X}-\left|\varepsilon_{B}\right|$. The (local) energy shift caused by the ir field and CL coupling in EA is given by

$$
\delta E_{\mathrm{COE}}^{\mathrm{EA}}(x, t)=\partial S_{k}^{\mathrm{EA}}(x, t) / \partial t
$$

and does not depend on the time-independent laser-free eikonal phase $S_{k}^{C}(x)$ in (16). For sub-fs xuv pulses, contributions to the time integral (19) mainly arise near the center of the xuv pulse at $t=-\tau$. Approximating $\delta E_{\mathrm{COE}}^{\mathrm{EA}}(x, \tau) \approx \partial S(x, t=-\tau) / \partial t$, we obtain

$$
\delta E_{\mathrm{COE}}^{\mathrm{EA}}(x, \tau)=-k A_{L}(\tau)+E_{C O E}^{\mathrm{CL}, 1}(x, \tau)+E_{\mathrm{COE}}^{\mathrm{CL}, 2}(x, \tau),
$$

where

$$
\begin{gathered}
E_{\mathrm{COE}}^{\mathrm{CL}, 1}(x, \tau)=\frac{U(x)}{k} A_{L}(\tau), \\
E_{\mathrm{COE}}^{\mathrm{CL}, 2}(x, \tau)=-\frac{1}{k} \int_{x}^{\infty} d x^{\prime} F\left(x^{\prime}\right) A_{L}\left(\frac{x^{\prime}-x}{k}-\tau\right)
\end{gathered}
$$

are the two contributions to the CL shift, $E_{\mathrm{COE}}^{\mathrm{CL}}=E_{\mathrm{COE}}^{\mathrm{CL}, 1}+$ $E_{\mathrm{COE}}^{\mathrm{CL}, 1}$, shown in Figs. 2(b) and 2(c) at $x=0$ for $\hbar \omega_{X}=90$ and $25 \mathrm{eV}$.

According to (22) and (23), $E_{\mathrm{COE}}^{\mathrm{CL}}(x, \tau)$ is proportional to $1 / k$, while $\delta E_{\mathrm{COE}}^{\mathrm{SFA}}(\tau)$ is proportional to $k$. Therefore, the CL coupling effect decreases for increasing PE kinitic energies. As shown in Figs. 2(b) and 2(c), the cancellation between $E_{\mathrm{COE}}^{\mathrm{CL}, 1}$ and $E_{\mathrm{COE}}^{\mathrm{CL}, 2}$ becomes stronger and further reduces the $\mathrm{CL}$ coupling with increasing $k$. Note that $E_{\mathrm{COE}}^{\mathrm{CL}, 1}(x, \tau)$ mainly increases the oscillation amplitude of the COE in SFA, while $E_{\mathrm{COE}}^{\mathrm{CL}, 2}(x, \tau)$ changes the oscillation amplitude and induces a phase shift. We can thus introduce a local temporal shift $\delta \tau(x)$ (relative to the SFA phase) and a local oscillation amplitude $K(x)$ by rewriting $(21)$ as

$$
\delta E_{\mathrm{COE}}^{\mathrm{EA}}(x, \tau)=K(x) A_{L}[\tau-\delta \tau(x)] .
$$
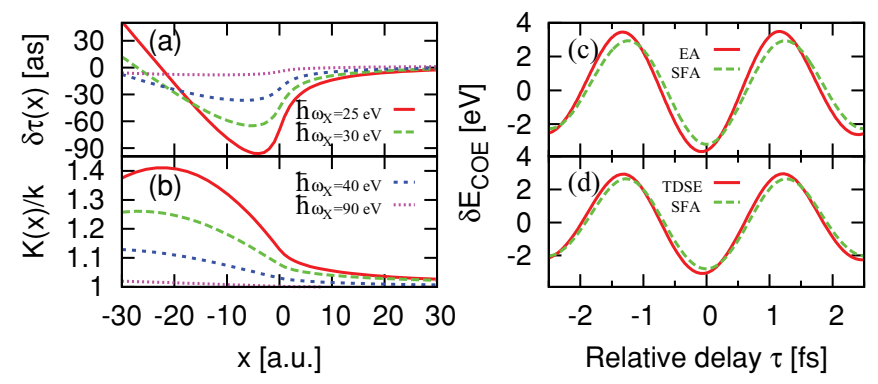

FIG. 3. (Color online) (a) Local temporal shift $\delta \tau(x)$ and (b) local oscillation amplitude $K(x)$ induced by the CL interaction in the EA. Comparison of the streaked COE obtained in the SFA with (c) the EA at $x=0$ and (d) the full TDSE calculations for $\hbar \omega_{X}=25 \mathrm{eV}$. The $\mathrm{PE}$ is assumed to move to the right $(k>0)$.

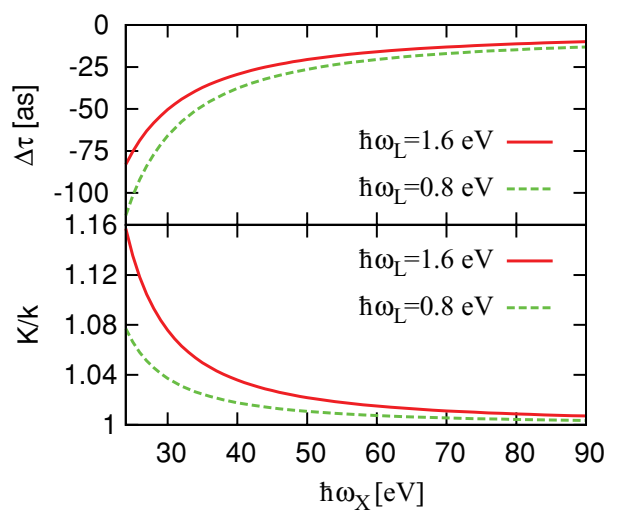

FIG. 4. (Color online) Comparison of eikonal temporal shift $\delta \tau$ and oscillation amplitude ratio $K / k$ at two $\omega_{L}$ values.

Figures 3(a) and 3(b) show $\delta \tau(x)$ and $K(x)$ as functions of $x$ for different $\omega_{X} . \delta \tau(x)$ can be positive or negative. The actual shift $\delta \tau$ in the streaking spectrum is obtained by spatial integration according to (19). However, as shown in Figs. 3(c) and $3(\mathrm{~d}), \delta E_{\mathrm{COE}}^{\mathrm{EA}}(x=0, \tau)$ agrees well with the TDSE result, since the initial wave function $\psi(x)$ is localized at $x=0$. Similarly, we find that the full TDSE results for $\delta \tau$ [solid line in Fig. 1(d) ] and $K / k$ [solid line in Fig. 1(e)] agree well with the EA results $\delta \tau(x)$ [dashed line in Fig. 1(d)] and $K(x) / k$ [dashed line in Fig. 1(e)] evaluated at $x=0$. This justifies approximating $\Delta S_{k}^{\mathrm{EA}}(x, t)=S_{k}^{\mathrm{EA}}(x, t)-S_{k}^{C}(x)$ in $(15)$,

$$
\begin{aligned}
\Delta S_{k}^{\mathrm{EA}}(x, t) & \approx S_{k}^{\mathrm{SFA}}(t)-\int_{t}^{\infty} d t^{\prime} F\left[x^{\prime}\left(t^{\prime}, t, x=0\right)\right] \Delta x\left(t, t^{\prime}\right) \\
& \approx-\frac{K E_{L}(t+\delta \tau)}{\omega_{L}^{2}}
\end{aligned}
$$

where, in the second line, the slow-varying envelope approximation is used.

We notice from (21)-(23) that the three contributions to $\delta E_{\mathrm{COE}}^{\mathrm{EA}}(x, \tau)$ are equally proportional to the ir electric field amplitude. Therefore, $\delta \tau$ and $K / k$ do not depend on the intensity of the ir pulse. However, upon reducing $\omega_{L},|\delta \tau|$ increases and $K / k$ decreases (Fig. 4). This is consistent with (23): at smaller $\omega_{L}, A_{L}$ oscillates more slowly, leading to less cancellation in the time integral and thus to larger $\delta \tau$. Simultaneously, stronger cancellation between $\delta E_{\mathrm{COE}}^{\mathrm{CL}, 1}$ and $\delta E_{\mathrm{COE}}^{\mathrm{CL}, 2}$ results in smaller $K(x)$.

\section{POLARIZATION OF THE INITIAL STATE BY THE IR PULSE}

The effect of initial-state polarization by the ir pulse on the streaked xuv photoemission spectrum has been addressed previously [19-21]. In this section, we analyze how it affects the temporal shift $\delta \tau$ and the oscillation amplitude ratio $K / k$. We find that the significance of the initial-state polarization depends on whether or not the laser unperturbed initial state is energetically isolated from other levels.

We first consider the nondegenerate case. For our 1D hydrogen atom, all levels are nondegenerate. In our TDSE calculation, the initial-state polarization by the ir pulse can be included (excluded) by keeping (dropping) the term $x E_{L}(t)$ 

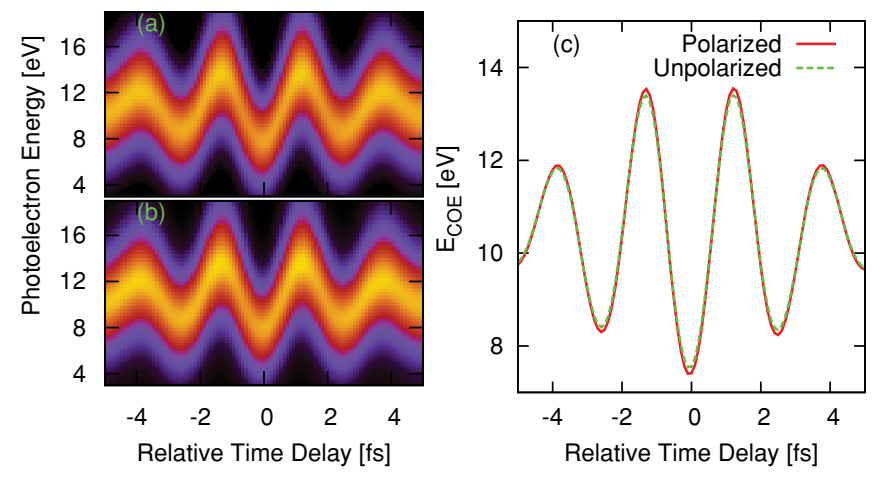

FIG. 5. (Color online) Polarization effect of the initial state on irstreaked photoemission from the ground state of 1D model hydrogen atoms for $\hbar \omega_{X}=25 \mathrm{eV}$. Spectrograms (a) with and (b) without initialstate polarization. (c) Corresponding centers of energies $\delta E_{\mathrm{COE}}(\tau)$.

in (2). In Figs. 5 and 6 we compare the polarized [Figs. 5(a) and 6(a)] and unpolarized [Figs. 5(b) and 6(b)] spectrograms and their corresponding centers of energy [Figs. 5(c) and 6(c)] for the ground-state level and the first excited state. Due to its large separation in energy from all excited states, the effect of the polarization of the ground state by the laser pulse on the spectrum is small. It slightly increases the oscillation amplitude but barely changes the temporal shift $\delta \tau$. In contrast, the first excited state, whose binding energy is $6.34 \mathrm{eV}$, can be easily polarized due to its laser-induced coupling to the second excited level at $3.64 \mathrm{eV}$. As shown in Fig. 6, the polarization distorts the spectrogram for negative delays where the ir pulse precedes the xuv pulse. This distortion does not uniformly shift the spectrograms. Therefore, $\delta \tau$ cannot be uniquely defined as a delay-independent temporal shift.

For the degenerate case, we consider a space spanned by the stationary wave functions $\psi_{200}(\mathbf{r})$ and $\psi_{210}(\mathbf{r})$ of the real (3D) hydrogen atom. Under the influence of the laser pulse, the wave function is

$$
\psi(\mathbf{r}, t)=\left[a_{200}(t) \psi_{200}(\mathbf{r})+a_{210}(t) \psi_{210}(\mathbf{r})\right] .
$$

By shifting the energy scale such that the binding energies of the two degenerate stationary states are $\varepsilon_{200}=\varepsilon_{210}=0$, and by substituting $\psi(\mathbf{r}, t)$ into the TDSE,

$$
i \frac{\partial}{\partial t} \psi(\mathbf{r}, t)=\left[H_{a t}+z E_{L}(t)\right] \psi(\mathbf{r}, t),
$$
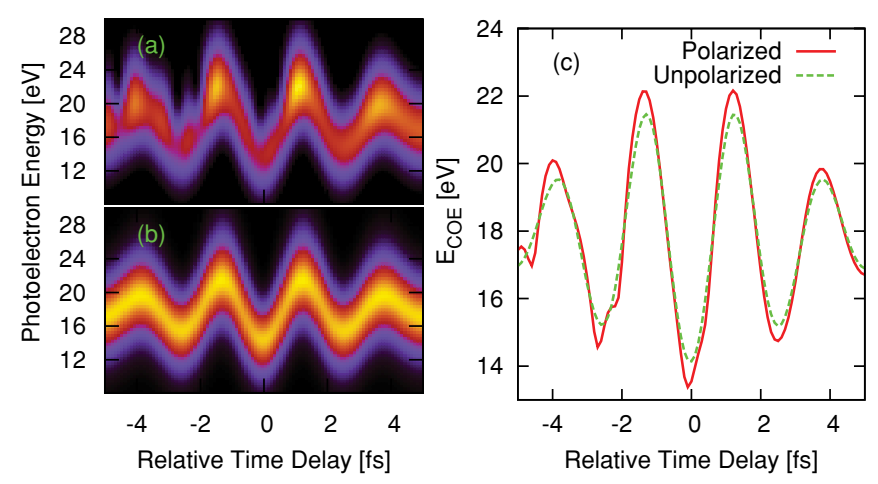

FIG. 6. (Color online) Same as Fig. 5, but for the first excited state of the 1D hydrogen atom. we obtain the equations of motion for the coefficients $a_{200}(t)$ and $a_{210}(t)$ :

$$
\begin{aligned}
i \frac{d}{d t} a_{200}(t) & =\mu E_{L}(t) a_{210}(t), \\
i \frac{d}{d t} a_{210}(t) & =\mu E_{L}(t) a_{200}(t),
\end{aligned}
$$

where $H_{\mathrm{at}}$ is the atomic Hamiltonian and $\mu=$ $\left\langle\psi_{200}(\mathbf{r})|z| \psi_{210}(\mathbf{r})\right\rangle$ the dipole-coupling matrix element. Equations (28) and (29) can be solved analytically [22]:

$$
\begin{aligned}
& a_{200}(t)=a_{200}^{0} \cos \left[\mu A_{L}(t)\right]+i a_{210}^{0} \sin \left[\mu A_{L}(t)\right], \\
& a_{210}(t)=a_{210}^{0} \cos \left[\mu A_{L}(t)\right]+i a_{200}^{0} \sin \left[\mu A_{L}(t)\right],
\end{aligned}
$$

where $a_{200}^{0}$ and $a_{210}^{0}$ are the initial amplitudes at $t_{0} \rightarrow-\infty$.

For example, the initial values $a_{200}^{0}= \pm 1 / \sqrt{2}$ and $a_{210}^{0}=$ $1 / \sqrt{2}$ give the wave functions

$$
\psi_{ \pm}(\mathbf{r}, t)=\psi_{ \pm}(\mathbf{r}) e^{ \pm i \mu A_{L}(t)}
$$

which evolve from the Stark states

$$
\psi_{ \pm}(\mathbf{r})=\frac{1}{\sqrt{2}}\left[\psi_{210}(\mathbf{r}) \pm \psi_{200}(\mathbf{r})\right]
$$

Similarly, for $a_{200}^{0}=1$ and $a_{210}^{0}=0$, we obtain the wave function

$$
\psi_{2 s}(\mathbf{r}, t)=\frac{1}{\sqrt{2}}\left[\psi_{+}(\mathbf{r}) e^{i \mu A_{L}(t)}-\psi_{-}(\mathbf{r}) e^{-i \mu A_{L}(t)}\right],
$$

which evolves from an initial $2 s$ state, while $a_{200}^{0}=0$ and $a_{210}^{0}=1$ results in a wave function that evolves from an stationary $2 p$ state:

$$
\psi_{2 p}(\mathbf{r}, t)=\frac{1}{\sqrt{2}}\left[\psi_{+}(\mathbf{r}) e^{i \mu A_{L}(t)}+\psi_{-}(\mathbf{r}) e^{-i \mu A_{L}(t)}\right] .
$$

Next, we calculate the ir-streaked spectrum using any of the wave functions (32)-(35) as the initial state in (18). To disentangle temporal shifts induced by (i) the initial-state polarization (relative to an unpolarized target) and (ii) the Coulomb potential acting on the final PE state (relative to the SFA; see Sec. III), we neglect the final-state distortion by the Coulomb potential and study initial-state polarization effects within the SFA. In this polarization-effect study, we hence use the Volkov wave function $\psi_{\mathbf{k}}(\mathbf{r}, t)$ as an approximation to the final state. If the initial state evolves from a stationary $2 s$ or $2 p$ state according to (34) or (35), the polarization causes delay-dependent interferences between the two Stark states $\psi_{ \pm}$. This interference significantly changes the energy-differential PE yield in the streaking trace in Fig. 7(b) relative to the trace for an unpolarized initial state in Fig. 7(a). However, the interference does not induce a relative temporal shift of the polarized relative to the unpolarized spectrum, which is best seen in the centers of energy of the two spectra in Fig. 7(c). This lack of an interference-induced temporal shift is explained by the fact that the dipole expectation values $\left\langle\psi_{2 s}(\mathbf{r}, t)|z| \psi_{2 s}(\mathbf{r}, t)\right\rangle$ and $\left\langle\psi_{2 p}(\mathbf{r}, t)|z| \psi_{2 p}(\mathbf{r}, t)\right\rangle$ are 0 at all times, even though the ir-laser pulse mixes the stationary $2 s$ and $2 p$ states. 

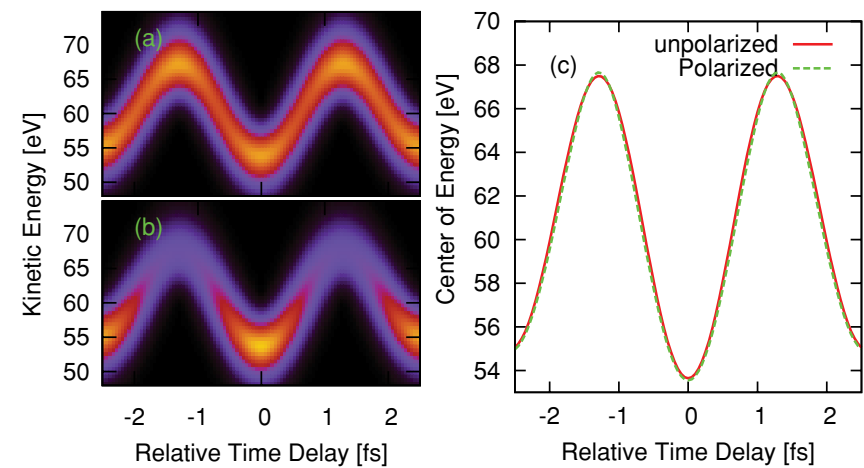

FIG. 7. (Color online) Photoelectron spectrum for an initial $2 s$ state of hydrogen: (a) neglecting ir-laser-induced initial-state polarization by setting $\mu=0$ in (34); (b) including laser-induced initial-state polarization, using $\mu=3$ a.u. in (34). (c) Corresponding centers of energies $E_{\mathrm{COE}}(\tau)$. No polarization-induced temporal shift is observed between the results for polarized and those for unpolarized initial states. Similar results (not shown) were obtained for initial $2 p$ states, using (35).

The situation is different for the states (32) that evolve out of the initial Stark states $\psi_{ \pm}$. Comparison of the energydifferential PE yields in the streaking trace for initial states (32) with and without inclusion of ir-laser-induced initial-state polarization shows only very small, hardly noticeable, differences [Figs. 8(a)-8(c)]. However, temporal shifts [21] become noticeable in the corresponding centers of energies. At a PE energy of $60 \mathrm{eV}$, they amount to 41 , as between an ir-laser-polarized and unpolarized initial $\psi_{+}$state [Fig. 8(d)], and to 82 , as between polarized initial $\psi_{+}$and $\psi_{-}$states [Fig. 8(e)]. These shifts originate in the permanent dipole
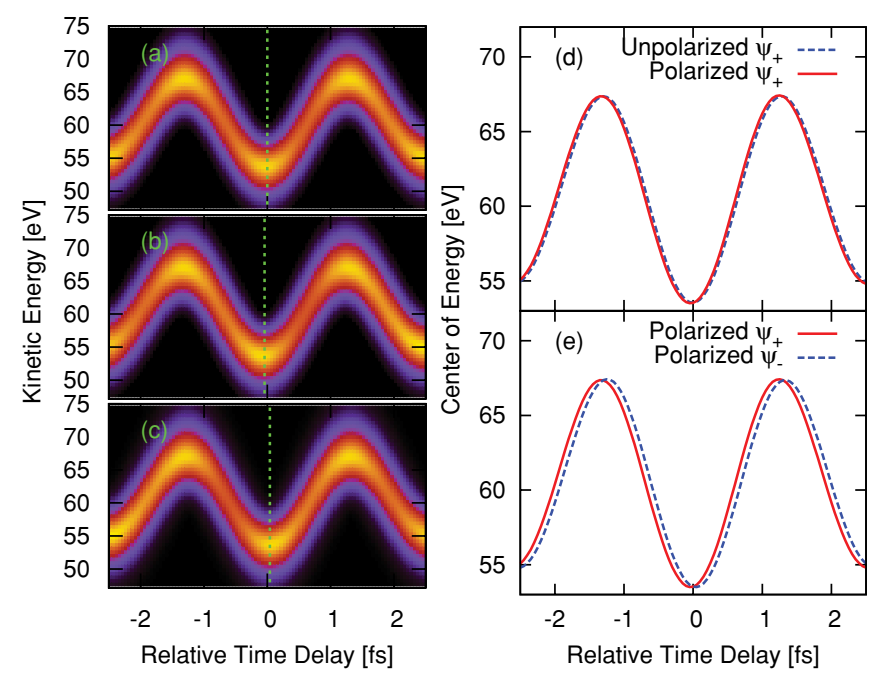

FIG. 8. (Color online) Photoelectron spectra for the initial $n=2$ Stark states (32) of hydrogen: (a) for $\psi_{+}(\mathbf{r}, t)$, neglecting ir-laserinduced initial-state polarization by setting $\mu=0$ in (32); (b) for $\psi_{+}(\mathbf{r}, t)$, including laser-induced initial-state polarization, using $\mu=$ 3 a.u. in (32); and (c) for $\psi_{-}(\mathbf{r}, t)$, including laser-induced initial-state polarization, using $\mu=3$ a.u. in (32). (d, e) Corresponding centers of energies $E_{C O E}(\tau)$, showing a relative temporal shift between the streaking traces of (d) polarized and unpolarized initial $\psi_{+}(\mathbf{r}, t)$ states and (e) polarized initial $\psi_{+}(\mathbf{r}, t)$ and $\psi_{-}(\mathbf{r}, t)$ states. moments of the Stark states whose interaction with the ir-laser electric field shifts the streaked spectra.

\section{CONCLUSIONS}

We have shown how the simultaneous interaction of an xuv PE with the electric field of a streaking ir-laser pulse and the Coulomb potential of the residual ion induces a specific CL coupling phase and leads to an attosecond temporal shift and amplitude enhancement in the oscillation of the streaked PE spectrum. This shift and amplitude enhancement become significant and observable as the xuv photon energy approaches the ionization threshold. It can be explained semiclassically in terms of an added Coulomb-phase factor in the PE wave function. This factor reveals the origin of the observable temporal shift as a CL coupling effect in the PE dynamics: the PE absorbs and releases ir photons while moving subject to the ionic Coulomb force. The analytical results obtained in the EA show that the CL coupling induces a temporal shift relative to $A_{L}$ and, thus, relative to the SFA result. For the experimental observation of $\delta \tau$ and $K / k$ as a function of the PE kinetic energy, we suggest using xuv pulses with tunable xuv photon energy to photoemit electrons from two levels with a large energy separation [23].

We have also examined the effect of ir-laser-induced polarization of the initial state on the ir-streaked xuv PE spectrum. If the initial state is not degenerate and has a large energetic separation from all other states, its very small polarization does not noticeably affect the PE spectrum. In contrast, if the initial state can easily be coupled to other states by the ir-laser pulse, its polarization is important and, interestingly, does not uniformly shift the spectrum. If the initial state has a permanent dipole moment, such as the $n=2$ Stark states of hydrogen, there is a relative temporal shift in the streaking traces (i) for different initial Stark states and (ii) with and without inclusion of the initial-state polarization.

\section{ACKNOWLEDGMENTS}

We thank F. He for helpful discussions. This work was supported by the NSF and the Division of Chemical Sciences, Office of Basic Energy Sciences, Office of Energy Research, US DOE. Some of the numerical computations for this project were performed on the Beocat cluster at Kansas State University.

\section{APPENDIX: USING THE EIKONAL WAVE FUNCTION FOR RABITT}

In this Appendix, we show that the atomic phase in RABITT and the relative temporal shift induced by the Coulomb interaction are identical within an EA. We start from the ir-assisted single-xuv-photon photoemission amplitude:

$$
T_{f i}(\tau)=-i \int_{-\infty}^{+\infty} d t\left\langle\psi_{f}(t)\left|\mathbf{r} \cdot \mathbf{E}_{\mathbf{X}}(t+\tau)\right| \psi_{i}(t)\right\rangle,
$$

where $\psi_{f}(t)$ and $\psi_{i}(t)$ are ir-dressed final and initial states, respectively. Differently from streaking, in RABITT, 
attosecond pulse trains (APTs) synthesized from a number of odd harmonics of the ir field,

$$
\mathbf{E}_{\mathbf{X}}(t)=\mathbf{E}_{\mathbf{X}, \mathbf{0}} \sum_{n} e^{-i \omega_{n} t+i \varphi_{n}}
$$

are used. Here $\omega_{n}=(2 n+1) \omega_{L}$ and $\varphi_{n}$ are the frequency and phase of the $(2 n+1)$ th harmonic, respectively, and $\omega_{L}$ is the fundamental frequency of the ir field. For simplicity, all harmonics are assumed to have the same strength $E_{X, 0}$. Using these APTs, we obtain a series of peaks in the PE spectrum that are separated by twice the ir-photon energy. In the presence of a weak ir field, sidebands will form between the main peaks due to the emission or absorption of ir photons. In RABITT periodic intensity variations are observed in the first sideband due to the inteference of two distinct two-photon transition routes: (1) absorption of one harmonic photon with frequency $\omega_{n}=(2 n+1) \omega_{L}$ and emission of an ir photon with frequency $\omega_{L}$ and (2) absorption of an adjacent lower harmonic photon with frequency $\omega_{n-1}=(2 n-1) \omega_{L}$ and an ir photon with frequency $\omega_{L}$. The intensity of the sideband is controlled by the delay $\tau$ between the APT and the ir field:

$$
P_{\mathrm{sb}}(\tau) \sim\left[1-\cos \left(2 \omega_{L} \tau+\Delta \varphi_{n}-\Delta \phi^{\mathrm{at}}\right)\right] .
$$

It is shifted by the harmonic phases $\Delta \varphi_{n}=\varphi_{n-1}-\varphi_{n}$ and the atomic phase $\Delta \phi^{\text {at }}[12,13,24] . \Delta \phi^{\text {at }}$ is a function of the PE energy.

Using the eikonal approximated wave function, we can now show that the atomic phase $\Delta \phi^{\text {at }} / 2 \omega_{L}$ is equal to the temporal shift $\delta \tau$ in streaked spectra. Using Eq. (25) for the EA phase $S_{k}^{\mathrm{EA}}$, expanding $\psi_{k}^{\mathrm{EA}}(x, t)$ up to first order in $E_{L}$,

$$
\begin{aligned}
\psi_{k}^{\mathrm{EA}}(x, t)= & \frac{1}{(2 \pi)^{1 / 2}} e^{i\left[k+A_{L}(t)\right] x} e^{-i E_{k} t+i S_{C}(x)} \\
& \times\left[1-\frac{i K E_{L}(t+\delta \tau)}{\omega_{L}^{2}}\right]
\end{aligned}
$$

substituting it into (A1) for $\psi_{f}$, and carrying out the time integration, we obtain the transition amplitude up to the twophoton process

$$
\begin{aligned}
T(\tau)= & -i d_{k} E_{X, 0} \sum_{n} \delta\left[E+I_{p}-(2 n+1) \omega_{L}\right] \\
& -i \frac{K d_{k}}{\omega_{L}^{2}} \frac{E_{L, 0} E_{X, 0}}{2} \sum_{n}\left\{\delta\left(E_{k}+I_{p}-2 n \omega_{L}\right) e^{-i \omega_{L}(\tau-\delta \tau)}\right. \\
& \left.-\delta\left[E_{k}+I_{p}-(2 n+2) \omega_{L}\right] e^{+i \omega_{L}(\tau-\delta \tau)}\right\},
\end{aligned}
$$

where $E_{k}=k^{2} / 2$ is the PE kinetic energy, $e^{-i\left(E_{k}+I_{p}\right) \tau}$ has been dropped,

$$
d_{k}=\frac{1}{(2 \pi)^{1 / 2}} \int d x e^{-i\left[k+A_{L}(t)\right] x} x e^{-i S_{C}(x)} \psi_{i}(x),
$$

and

$$
\begin{aligned}
& \tilde{E}_{L}(\omega)=\frac{E_{L, 0}}{2 i}\left[\delta\left(\omega+\omega_{L}\right)-\delta\left(\omega-\omega_{L}\right)\right], \\
& \tilde{E}_{X}(\omega)=\sum_{n} E_{X, 0} e^{+i \varphi_{n}} \delta\left[\omega-(2 n+1) \omega_{L}\right]
\end{aligned}
$$

are the Fourier transformations of $E_{L}(t)$ and $E_{X}(t)$. The transition amplitude for sidebands at PE energies $E_{k}=2 n \omega_{L}-I_{p}$ follows as

$$
\begin{aligned}
T_{\mathrm{sb}}\left(E_{f}, \tau\right)= & -i \frac{K d_{k} E_{L, 0}}{2 \omega_{L}^{2}} E_{X, 0} e^{-i \varphi_{n}-i \omega_{L}(\tau-\delta \tau)} \\
& \times\left[1-e^{i \Delta \varphi_{n}+2 i \omega_{L}(\tau-\delta \tau)}\right] .
\end{aligned}
$$

Accordingly, the sideband intensity as a function of delay $\tau$ is

$$
\begin{aligned}
P_{\mathrm{sb}}\left(E_{k}, \tau\right)= & \left|T_{s b}(\tau)\right|^{2}=\frac{K^{2} d_{k}^{2} E_{L, 0}^{2} E_{X, 0}^{2}}{2 \omega_{L}^{4}} \\
& \times\left\{1-\cos \left[2 \omega_{L}(\tau-\delta \tau)+\Delta \varphi_{n}\right]\right\},
\end{aligned}
$$

from which the atomic phase $\Delta \phi^{\text {at }} / 2 \omega_{L}=\delta \tau$ is identified.
[1] F. Krausz and M. Ivanov, Rev. Mod. Phys. 81, 163 (2009), and references therein.

[2] M. Y. Ivanov, M. Spanner, and O. Smirnova, J. Mod. Opt. 52, 165 (2005).

[3] C.-H. Zhang and U. Thumm, Phys. Rev. Lett. 102, 123601 (2009).

[4] A. L. Cavalieri et al., Nature 449, 1029 (2007).

[5] A. K. Kazansky and P. M. Echenique, Phys. Rev. Lett. 102, 177401 (2009).

[6] We thank A. L. Cavalieri and N. Karpowicz for sharing their unpublished experimental data.

[7] S. Varró and F. Ehlotzky, J. Phys. B 31, 2145 (1998).

[8] C. Lemell, B. Solleder, K. Tökési, and J. Burgdörfer, Phys. Rev. A 79, 062901 (2009).

[9] J. C. Baggesen and L. B. Madsen, Phys. Rev. A 78, 032903 (2008); 80, 030901(R) (2009).
[10] N. M. Kroll and K. M. Watson, Phys. Rev. A 8, 804 (1973).

[11] O. Smirnova, M. Spanner, and M. Ivanov, Phys. Rev. A 77, 033407 (2008); J. Phys. B 40, F197 (2007).

[12] V. Veniard, R. Taïeb, and A. Maquet, Phys. Rev. A 54, 721 (1996).

[13] E. S. Toma and H. G. Muller, J. Phys. B 35, 3435 (2002).

[14] J. Mauritsson, M. B. Gaarde, and K. J. Schafer, Phys. Rev. A 72, 013401 (2005).

[15] K. Varjú, P. Johnsson, R. López-Martens, T. Remetter, E. Gustafsson, J. Mauritsson, M. B. Gaarde, K. J. Schafer, Ch. Erny, I. Sola, A. Zaïr, E. Constant, E. Cormier, E. Mével, and A. L'Huillier, Laser Phys. 15, 888 (2005).

[16] C. Joachain, Quantum Collision Theory (North-Holland Publish Company, Amsterdam, 1983).

[17] J. I. Gersten and M. H. Mittleman, Phys. Rev. A 12, 1840 (1975). [18] U. Thumm, J. Phys. B 25, 421 (1992), and references therein. 
[19] A. K. Kazansky and N. M. Kabachnik, J. Phys. B 40, 2163 (2007).

[20] O. Smirnova, A. S. Mouritzen, S. Patchkovskii, and M. Ivanov, J. Phys. B 39, S323 (2006).

[21] J. C. Baggesen and L. B. Madsen, Phys. Rev. Lett. 104, 043602 (2010).
[22] F. Grossmann, Theoretical Femtosecond Physics (Springer, Berlin, 2008).

[23] M. Schultze et al., Science 328, 1658 (2010).

[24] P. M. Paul, E. S. Toma, P. Breger, G. Mullot, F. Augé, Ph. Balcou, H. G. Muller, and P. Agostini, Science 292, 1689 (2001). 